\title{
Electrode Fabrication, Material Characterization and Capacitance Measurement Studies of Polyacrylonitrile/Polypyrrole(PAN/PPY) Composite
}

\author{
Mohammad Faraz Ahmer ${ }^{1, *}$, Salman Hameed ${ }^{2}$ \\ 1 Assistant Professor, Department of Electrical and Electronics Engineering, Mewat Engineering College, Haryana, India; \\ farazahmer007@gmail.com; \\ 2 Associate Professor, Department of Electrical Engineering, Faculty of Engineering and Technology, Aligarh Muslim \\ University, Aligarh, India; hameeddee@gmail.com; \\ * Correspondence: farazahmer007@gmail.com;
}

Received: 30.05.2020; Revised: 20.06.2020; Accepted: 22.06.2020; Published: 25.06.2020

\begin{abstract}
For the fabrication of the electrode, the Polyvinyl alcohol/polyaniline composite was prepared by the sol-gel method. The electrical conductivity of the composite was determined on compressed pellets by using a 4-in-line-probe dc electrical conductivity-measuring instrument. The electrical conductivity measurement studies revealed that the composite possessed the electrical conductivity in the range of 10-4 to 10-2 S cm-1, i.e., in the semiconductor region and followed the Arrhenius equation. The thermal stability of the composite material ( $\mathrm{HCl}$ treated) in terms of dc electrical conductivity retention was studied under isothermal conditions (at 50,70, 90, 110, 130, and $150{ }^{\circ} \mathrm{C}$ ) at $15 \mathrm{~min}$ intervals. The stability of the material ( $\mathrm{HCl}$ treated) in terms of electrical conductivity retention was also monitored for five cycles at increasing temperatures with $1 \mathrm{~h}$ intervals. The composite material was found thermally and environmentally stable in terms of dc electrical conductivity retention.
\end{abstract}

Keywords: Electrode fabrication; characterization; thermal stability; organic/organic composite; polyvinyl alcohol/polyaniline.

(c) 2020 by the authors. This article is an open-access article distributed under the terms and conditions of the Creative Commons Attribution (CC BY) license (https://creativecommons.org/licenses/by/4.0/).

\section{Introduction}

Supercapacitors are known as electrochemical storage devices that can store the energy and power densities to complement the function of batteries and conventional capacitors for storing energy and delivering power [1]. For the development of electrochemical devices, various types of electrode materials have been extensively investigated because the property of the electrode materials directly influences the performance of supercapacitors. However, activated carbon is one of the most important conventional supercapacitor electrode materials $[2,3]$. It has been significantly used due to its low cost, large capacitance, and long cycling life [4]. However, the energy storage mechanism of activated carbon is through a double-layer process, resulting in lower energy density and specific capacitance comparing with the energy storage mechanism in redox reaction [5]. Therefore, some of the conducting polymers like polyaniline and polypyrrole have also been used because of their high conductivity, low cost, easy polymerization, and reversible doping/deduping property [6-10]. However, these intrinsically conducting polymers have several disadvantages of low processability and mechanical stability. To solve these problems, conducting polymers of various conducting composites have been synthesized and studied by several researchers [11]. In the present work, 
polyacrylonitrile/polypyrrole (PAN/Ppy) composite was synthesized, and the structure, composition and electrochemical performance were examined.

\section{Materials and Methods}

The reagents used for the synthesis of the material were obtained from CDH, Loba Chemie, E- Merck, and Qualigens (India Ltd.). All other reagents and chemicals were of analytical reagent grade. A four-in-line probe electrical conductivity-measuring instrument, Scientific Equipment (India), was used for measuring the dc electrical conductivity. A hydraulic pressure instrument was used for making pellets of sample materials. An electronic balance (digital), Sartorius (Japan), model 21 OS was used for weighing purposes.

\subsection{Preparation of composite material.}

Various samples of polyacrylonitrile/polypyrrole (PAN/Ppy) were prepared by mixing one volume of 5 weight $\%$ solution of polyacrylonitrile solution with different volumes of pyrrole (approximately $3.33 \mathrm{wt} \%$ solutions in toluene) as given in Table 1 . FeCl3 solution $(0.1$ M) was mixed thoroughly with the PAN/Pyrrole. Continuous stirring was done during the addition of $\mathrm{FeCl} 3$ solution, slowly the white gel of precipitate gel PAN/Ppy turned first to green and then to black. The reaction mixture was kept for $24 \mathrm{~h}$ under ambient condition $\left(25 \pm 2{ }^{\circ} \mathrm{C}\right)$. The reaction mixture was then kept for $24 \mathrm{~h}$ under ambient conditions $\left(25 \pm 2{ }^{\circ} \mathrm{C}\right)$. Then the polypyrrole based composite gel was filtered off, washed with $0.75 \mathrm{M} \mathrm{HCl}$ and then washed thoroughly with DMW to remove excess acids and any adhering traces of ferric chloride. After filtration, the gel was dried at $50{ }^{\circ} \mathrm{C}$ in an air oven for $48 \mathrm{~h}$. The dry product was then crushed into small granules when immersed in DMW. The material was again washed with acetone in a soxhlet, finally dried at $50{ }^{\circ} \mathrm{C}$ and kept in a desiccator. Hence a number of composite samples of PAN/Ppy were prepared.

\subsection{Preparation of composite pellet.}

The polyacrylonitrile/polypyrrole (PAN/Ppy) composite material and polystyrene were ground into fine powder separately using mortar pestle and sieved through 200 mesh (granule size $<0.07 \mathrm{~mm})$ sieve. The composite material $(0.5 \mathrm{~g})$ was mixed with $0.2 \mathrm{~g}$ of polystyrene with the help of mortar and pestle. The pellets of this material were made at room temperature with the help of a hydraulic pressure instrument at $25 \mathrm{KN}$ pressure for $20 \mathrm{~min}$. The pellet casting die was placed in an oven maintained at $200{ }^{\circ} \mathrm{C}$ for about $1 \mathrm{~h}$ to equilibrate the reaction mixture [12].

Table 1. Conditions of the preparation of PAN/Ppy composite.

\begin{tabular}{l|l|l|l|l|l} 
Samples & \multicolumn{4}{l}{ Mixing volume ratio (v/v) } \\
\cline { 2 - 6 } $\begin{array}{l}\text { S-1 } \\
\text { S-2 }\end{array}$ & PAN 5\% in DMF & $\begin{array}{l}\mathrm{FeCl} 3(1 \mathrm{M} \text { in } 1 \text { M } \\
\mathrm{HCl})\end{array}$ & $\begin{array}{l}\text { Pyrrole in toluene } \\
(33.33 \%)\end{array}$ & $\begin{array}{l}\text { Appearance of beads } \\
\text { after drying }\end{array}$ \\
\cline { 2 - 6 } S-3 & 1 & 1 & 1 & Black \\
\hline S-4 & 1 & 1 & 2 & Black \\
\cline { 2 - 6 } S-5 & 1 & 1 & 3 & Black \\
\cline { 2 - 6 } & 1 & 1 & 5 & Black
\end{tabular}

2.3. $X$-ray diffraction $(X R D)$ study.

X-ray diffraction pattern of polyacrylonitrile/polypyrrole (PAN/Ppy) composite material was recorded by Miniflex-II, X-Ray diffractometer (Rigaku Corporation) with $\mathrm{Cu} \mathrm{K \alpha}$ radiation. 


\subsection{Fourier transform infrared (FTIR) spectroscopic study.}

The FTIR spectrum of polyacrylonitrile/polypyrrole (PAN/Ppy) composite material was recorded by the Interspec-20 FTIR spectrometer, Spectrolab (U.K.). The sample compartment was $200 \mathrm{~mm}$ wide, $290 \mathrm{~mm}$ deep, and $255 \mathrm{~mm}$ high. The entrance and exit beam to the sample compartment were sealed with a coated $\mathrm{KBr}$ window, and there was a hinged cover to seal it from the environment.

2.5. Thermogravimetric analysis (TGA)/differential thermal analysis (DTA)/ differential thermogravimetric analysis (DTG).

The degradation process and thermal stability of polyacrylonitrile/polypyrrole (PAN/Ppy) composite were investigated using thermogravimetric analysis (TGA) (Exstar TG/DTA-6300), under a nitrogen atmosphere using a heating rate of $20{ }^{\circ} \mathrm{C}$ per min from 28 to $800{ }^{\circ} \mathrm{C}$.

\subsection{Scanning electron microscopic (SEM) studies.}

Scanning electron microscopic images of polyacrylonitrile/polypyrrole (PAN/Ppy) composite was taken by JEOL, JSM, 6510-LV, (Japan) scanning electron microscope at an accelerating voltage of $20 \mathrm{kV}$. The sample was mounted on a copper stub and sputter-coated with gold to minimize the charging.

\subsection{Energy dispersive $x$-ray analysis (EDX) studies.}

Energy-dispersive X-ray analysis studies were performed to get the elemental composition of polyacrylonitrile/polypyrrole (PAN/Ppy) composite while taking scanning electron microphotographs of chemically prepared polyacrylonitrile/polypyrrole (PAN/Ppy) composite by JEOL, JSM, 6510-LV, (Japan) scanning electron microscope.

\subsection{Measurement of capacitance.}

The capacitance of polyacrylonitrile/polypyrrole (PAN/Ppy) composite was evaluated by cyclic voltammetry in $0.1 \mathrm{M} \mathrm{NaClO} 4$ aqueous electrolyte within the potential range of $-3.0 \mathrm{~V}$ to $3.0 \mathrm{~V}$ at various scan rates. The capacitance characteristics of the polyacrylonitrile/polypyrrole (PAN/Ppy) composite was carried out in $0.1 \mathrm{M} \mathrm{NaClO} 4$ aqueous solution in a three-electrode cell, as mentioned above. The specific capacitance can be obtained from the $\mathrm{CV}$ curves by the following equation:

$$
C_{\mathrm{sp}}=\frac{i}{\mathrm{sm}}\left(\mathrm{F} \mathrm{g}^{-1}\right)
$$

Here i present the average cathodic current; $\mathrm{s}$ and $\mathrm{m}$ present the scanning rate and the mass of the electrode, respectively [13].

\section{Results and Discussion}

In this study, various samples of polyacrylonitrile/polypyrrole (PAN/Ppy) composite materials were prepared by the incorporation of polypyrrole into the matrices of PAN by mixing different concentrations (vol. \%) of organic monomers pyrrole into the fixed volume of PAN solution. The variation of ambient temperature on electrical conductivity $(\sigma)$ of PAN/Ppy 
composite samples was carried out, and sample (S-3) having high electrical conductivity was selected for further studies (Table 1). The X-ray diffraction analysis (Fig. 1) showed very strong peaks to confirm the crystalline nature of composite material and supporting the ordered arrangement of molecules.

FTIR spectra for the PAN/Ppy composite is shown in Fig. 2. A broadband dip around $3500 \mathrm{~cm}^{-1}$ is expected for the N-H stretching vibrations $[14,15]$. The band at $1557.39 \mathrm{~cm}-1$ is related to $\mathrm{C}=\mathrm{C} / \mathrm{C}-\mathrm{C}$ stretching vibrations in the pyrrole rings. The band at approximately $1452.43 \mathrm{~cm}-1$ reflects $\mathrm{C}-\mathrm{N}$ stretching vibrations in the rings. The peak at $1316.38 \mathrm{~cm}-1$ is attributed to $\mathrm{C}-\mathrm{N}$ in-plane deformation modes, and a band related to $\mathrm{C}-\mathrm{H}$ in-plane deformation vibrations is shown at $1046.10 \mathrm{~cm}-1$. The bands at 927.22 and $789.72 \mathrm{~cm}-1$ are related to the C-H out-of-plane deformation vibrations of the polymer rings. The peak at $685.05 \mathrm{~cm}-1$ may be related to $\mathrm{C}-\mathrm{C}$ out-of-plane ring deformation vibrations or $\mathrm{C}-\mathrm{H}$ rocking. The positions of different peaks in the spectrum indicate the formation of composite by the combination of constituting components.



Figure 1. X-ray diffraction pattern of polyacrylonitrile/polypyrrole (PAN/Ppy) composite material.

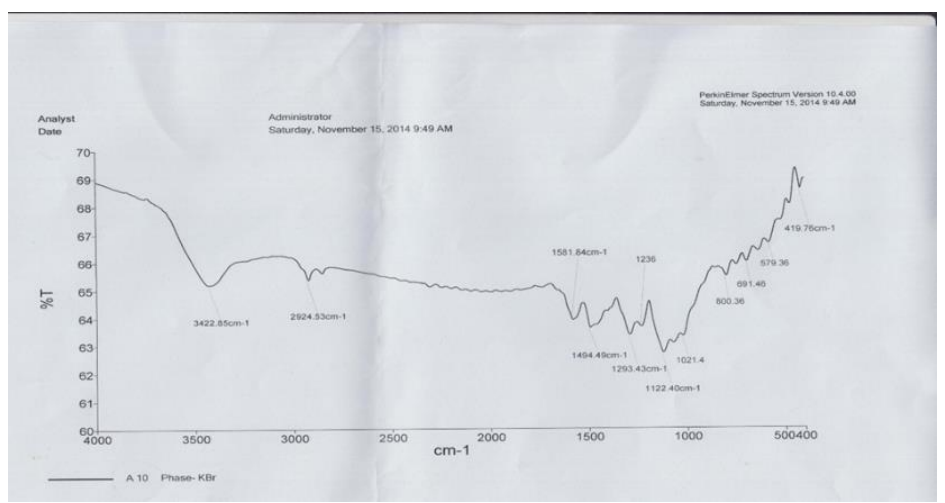

Figure 2. FTIR spectra of polyacrylonitrile/polypyrrole (PAN/Ppy) composite material.

The energy dispersive X-ray analysis showed the presence of $\mathrm{C}, \mathrm{N}, \mathrm{O}$ confirming the formation of polyacrylonitrile/polypyrrole (PAN/Ppy) composite (Fig. 3). The weight percent elemental composition of the constituents of the composite is as $\mathrm{C}=61.63 \%, \mathrm{~N}=27.12$, $\mathrm{O}=13.84$. 
The SEM image of polyacrylonitrile/polypyrrole (PAN/Ppy) composite material is shown in Fig. 4. The scanning electron micrograph of polyacrylonitrile/polypyrrole (PAN/Ppy) composite material showed uniform granular surface morphology.

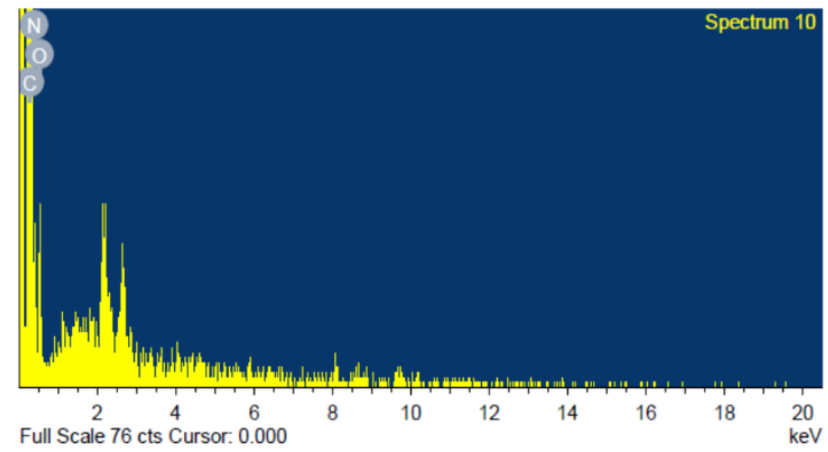

Figure 3. X-ray photoelectron spectra of polyacrylonitrile/polypyrrole (PAN/Ppy) composite material.

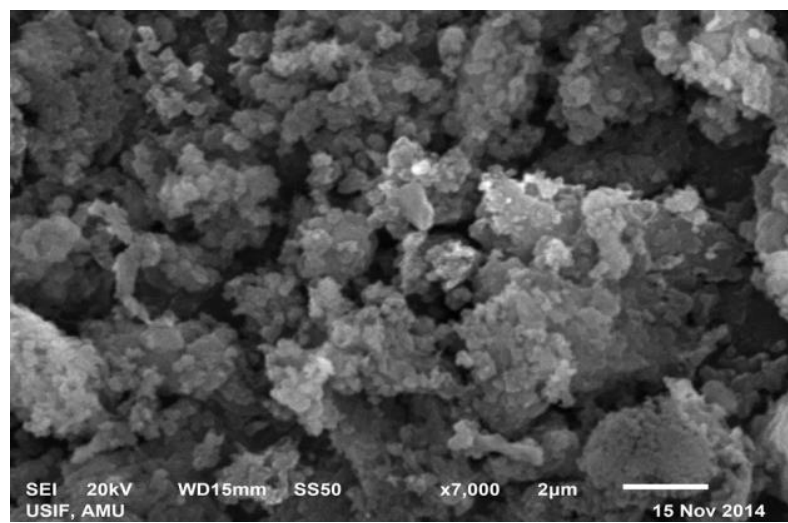

Figure 4. The SEM image of polyacrylonitrile/polypyrrole (PAN/Ppy) composite material.


Figure 5. Cyclic voltammetry curves in $0.1 \mathrm{M} \mathrm{NaClO}_{4}$ with different scan rates (a) $0.025 \mathrm{~V} \mathrm{~s}-1$, (b) $0.5 \mathrm{~V} \mathrm{~s}-1$ and $0.1 \mathrm{~V} \mathrm{~s}-1$ for polyacrylonitrile/polypyrrole (PAN/Ppy) composite material electrode.

It can be seen form cyclic voltammetry curves that the composite showed redox cyclability at each scan rate (Fig. 5 a-c). It is also clear from the graphs that the composite showed the capacitive cyclic voltammetry curve [13]. The current density is increasing with the increase in the scan rate, and a maximum of the anodic and cathodic current density of 248 and 149 A g-1, respectively, was achieved during cyclic voltammetry. The specific capacitance was also calculated from the cyclic voltammetry curve using the above-mentioned formula and was found to be $222 \mathrm{~F}$ g-1. Fig. 6 showed the galvanic charge/discharge curves of polypyrrole/PAN composite in a three-electrode system. It is clear from the figure that as the charge/discharge current density increases from 0 A g-1 upto 222 A g-1, the specific capacitance of the electrode is retained for about $95 \%$. This retention is the specific capacitance 
of the electrode may be due to high redox cyclability of the electrode may be due to the presence of polypyrrole in polypyrrole/PAN composite.

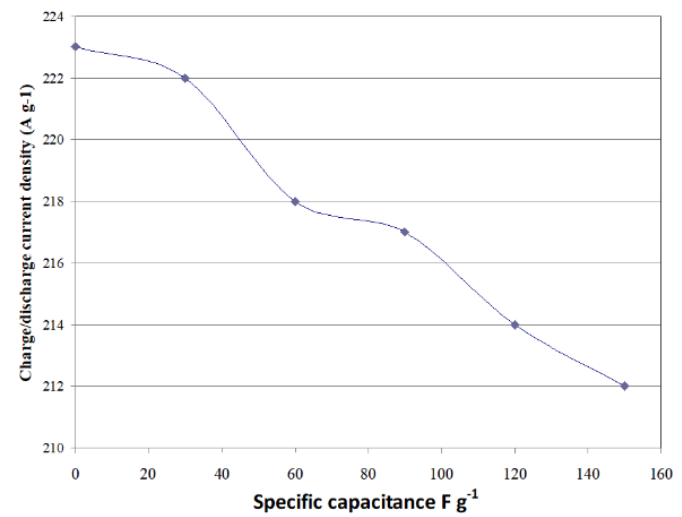

Figure 6. Charge/discharge curve of polyacrylonitrile/polypyrrole (PAN/Ppy) composite electrode at different current density.

\section{Conclusions}

In this study, a new polyacrylonitrile/polypyrrole (PAN/Ppy) composite was prepared and characterized by instrumental and electrochemical methods. The electrochemical characteristics of the composite showed good capacitive properties. The galvanic chargedischarge studies showed good capacitance retention at different current densities.

\section{Funding}

This research received no external funding.

\section{Acknowledgments}

The authors are thankful to the Department of Electrical Engineering, Sri Venkateswara University, and Aligarh Muslim University, for providing necessary research facilities.

\section{Conflicts of Interest}

The authors declare no conflict of interest.

\section{References}

1. Liu, Y.; Hu, Z.; Xu, K.; Zheng, X.; Gao, Q. Surface Modification and Performance of Activated Carbon Electrode Material. Acta Physico-Chimica Sinica 2008, 24, 1143-1148, https://doi.org/10.1016/S18721508(08)60049-2.

2. Chen, W.C.; Wen, T.C.; Teng, H. Polyaniline-deposited porous carbon electrode for supercapacitor. Electrochimica Acta 2003, 48, 641-649,], https://doi.org/10.1016/S0013-4686(02)00734-X.

3. Jia, Y.; Jiang, J.; Sun, K.; Dai, T. Enhancement of capacitance performance of activated carbon-Polyaniline composites by introducing methyl orange. Electrochimica Acta 2012, 71, 213-218, https://doi.org/10.1016/j.electacta.2012.03.150.

4. Bleda-Martínez, M.J.; Morallón, E.; Cazorla-Amorós, D. Polyaniline/porous carbon electrodes by chemical polymerisation: Effect of carbon surface chemistry. Electrochimica Acta 2007, 52, 4962-4968, https://doi.org/10.1016/j.electacta.2007.01.073.

5. Ko, J.M.; Song, R.Y.; Yu, H.J.; Yoon, J.W.; Min, B.G.; Kim, D.W. Capacitive performance of the composite electrodes consisted of polyaniline and activated carbons powder in a solid-like acid gel electrolyte. Electrochimica Acta 2004, 50, 873-876,https://doi.org/10.1016/j.electacta.2004.02.064.

6. Salinas-Torres, D.; Sieben, J.M.; Lozano-Castello, D.; Morallón, E.; Burghammer, M.; Riekel, C.; CazorlaAmorós, D. Characterization of activated carbon fiber/polyaniline materials by position-resolved microbeam small-angle X-ray scattering. Carbon 2012, 50, 1051-1056,https://doi.org/10.1016/j.carbon.2011.10.010. 
7. Dalmolin, C.; Biaggio, S.R.; Rocha-Filho, R.C.; Bocchi, N. Preparation, electrochemical characterization and charge-discharge of reticulated vitreous carbon/polyaniline composite electrodes. Electrochimica Acta 2009, 55, 227-233,https://doi.org/10.1016/j.electacta.2009.08.043.

8. Ramamurthy, P.C.; Malshe, A.M.; Harrell, W.R.; Gregory, R.V.; McGuire, K.; Rao, A.M.8. Handbook of Multiphase Polymer Systems.Solid State Electronics2004,48, 2019-2024.

9. Wang, Z.; Zhu, Z.-Z.; Shi, J.; Li, H.-L. Electrocatalytic oxidation of formaldehyde on platinum welldispersed into single-wall carbon nanotube/polyaniline composite film. Applied Surface Science 2007, 253, 8811-8817, https://doi.org/10.1016/j.apsusc.2007.03.005.

10. Zhao, X.; Lv, L.; Pan, B.; Zhang, W.; Zhang, S.; Zhang, Q. Polymer-supported nanocomposites for environmental application: A review. Chemical Engineering Journal 2011, 170, 381-394, https://doi.org/10.1016/j.cej.2011.02.071.

11. Woo, S.W.; Dokko, K.; Nakano, H.; Kanamura, K. Incorporation of polyaniline into macropores of threedimensionally ordered macroporous carbon electrode for electrochemical capacitors. Journal of Power Sources 2009, 190, 596-600, https://doi.org/10.1016/j.jpowsour.2009.01.050.

12. Khan, M.M.A.; Rafiuddin; Inamuddin; Naushad, M. Synthesis, surface characterization and electrochemical properties of PVC-based cerium(IV) sulphate ion exchange composite membrane. Ionics 2015, 21, 10571062,https://doi.org/10.1007/s11581-014-1246-5.

13. Hu, Y.; Zhao, Y.; Li, Y.; Li, H.; Shao, H.; Qu, L. Defective super-long carbon nanotubes and polypyrrole composite for high-performance supercapacitor electrodes. Electrochimica Acta 2012, 66, 279-286, https://doi.org/10.1016/j.electacta.2012.01.093.

14. Chougule, M.A.;Pawar, S.G.; Godse, P.R.;Mulik, R.N.;Patil, V.B.; Sen, S. Synthesis and characterisation of Polypyrrole (Ppy) thin films. S. Soft Nanoscience Letter 2011, 1, 6-10, https://doi.org/10.4236/snl.2011.11002.

15. Su, N.; Li, H.B.; Yuan, S.J.; Yi, S.P.; Yin, E.Q. Synthesis and characterization of polypyrrole doped with anionic spherical polyelectrolyte brushes Express Polymer Letters 2012, 6, 697705,https://doi.org/10.3144/expresspolymlett.2012.75. 